\title{
Measuring the Energy Landscape in Single Semiconductor Nanowires
}

\author{
L.M. Smith ${ }^{a}$, H.E. JaCKSON ${ }^{a}$, J. YARRison-Rice ${ }^{b}$ And Ch. JAGAdish ${ }^{c}$
}

${ }^{a}$ Department of Physics, University of Cincinnati, Cincinnati, Ohio 45221-0011, United States

${ }^{b}$ Department of Physics, Miami University, Oxford, Ohio 45056, United States

${ }^{c}$ Department of Electronic Materials Engineering, Research School of Physics and Engineering

The Australian National University, Canberra, ACT 0200, Australia

With the ability to design and control the physical structure of nanostructures to tune their electronic properties, it is increasingly important to measure the electronic structure of single nanostructures. Here we describe a number of experimental techniques for measuring the electronic structure of single semiconductor nanowires. The advantages, disadvantages and limitations of these methods will be described.

PACS: 73.22.-f, 73.43.Fj, 73.50.Gr, 73.50.Pz, 73.63.-b, 78.30.Fs, 78.35.+c, 78.55.-m, 78.67.-n

\section{Introduction}

In recent years, significant efforts have been made to develop methods of tuning the electronic structure of materials by controlling their physical structure on the nanoscale [1-6]. These trends will only accelerate in the future as hybrid materials come to the forefront where semiconductor, metallic, organic or biomolecular materials are combined into nanoscale structures which can be designed for particular purposes in basic sciences or in technology [7]. The fundamental problem is that as the length scale of the control decreases, the sensitivity to variation between nanostructure to nanostructure becomes ever larger and thus there is intense interest in developing methods for measuring the electronic landscape in single nanostructures.

This paper will review a number of methods used to study the electronic structure in single semiconductor nanowire heterostructures using optical techniques. Photoluminescence, time-resolved photoluminescence, photoluminescence excitation, the Raman scattering, resonant Raman scattering, photocurrent spectroscopy and photomodulated Rayleigh scattering spectroscopy will be discussed with an eye to enumerating both their strengths and weaknesses.

\section{Materials}

Semiconductor nanowires and nanosheets are important examples of nanostructures whose understanding benefits from these experimental developments. Both nanosheets and nanowires can be grown using vaporliquid-solid growth techniques where a metallic (usually gold) nanoparticle is used as a catalyst of the quasi-one-dimensional or two-dimensional growth [1, 2, 5]. Most importantly, by changing the source material and the parameters of the growth it is possible to grow both radial or axial heterostructures between almost any two materials, regardless of the degree of lattice mismatch [8-11]. Many materials systems also exhibit polytypism between wurtzite (WZ, hexagonal) or zinc blende (ZB, cubic) symmetries which results in important changes in both the valence and conduction bands [4, 12-14]. Through the control of all of these processes it is possible to tune the energy landscape of nanowire heterostruc- tures through quantum confinement in either the radial or axial directions, through the relative strain of the core and shell, and finally through control of the $\mathrm{ZB}$ and $\mathrm{WZ}$ polytypes. Studying such structures requires detailed information on exactly how the energy landscape changes in particular structures, as is discussed for a number of specific cases below.

\section{Photoluminescence}

The technique of photoluminescence (PL) is both powerful and also the standard initial method for characterizing semiconductor nanowires. A laser which emits photons with energy larger than the energy gap is used to excite electron-hole pairs high up into the valence and conduction bands of a semiconductor nanostructure. The electrons and holes rapidly thermalize within a picosecond to the lowest energy states within the nanostructure, and may then emit light which is characteristic of this final state [15]. Using a high quality microscope objective one can couple light which is emitted from a single nanostructure (or even from a small section of a nanowire) to a spectrometer and imaging detector. Through such techniques one gains information on the ground state energy of the nanostructure with sub-meV energy resolution and with micron or sub-micron spatial resolution [16-18].

To obtain information on excited states, one can use excitation power-dependent measurements so as to saturate the ground states and begin to populate the excited states. For example, in $\mathrm{WZ} / \mathrm{ZB}$ InP nanowires power dependent measurements showed that the confined electronic states in the $\mathrm{ZB}$ sections and hole states in the WZ sections could be easily saturated so that the emitted PL spectral energies could be tuned over a hundred milli-electron volts $[19,20]$. Similar power-dependent measurements by van Kouwen et al. in InAsP quantum dots confined within InP nanowires showed clear evidence of the confined excited structure of the dot [21]. Density dependent measurements can also be accomplished through the use of time-resolved PL (TRPL) where the electronhole pair density can be effectively varied over orders of magnitude by collecting PL spectra as a function of time after pulsed excitation allowing the electron-hole pairs 
to decay [22]. For example, TRPL measurements were used to map out the energy structure in mixed-phase $\mathrm{WZ} / \mathrm{ZB}$ InP nanowires, and in fact confirmed the type-II band alignments since the more strongly confined electron and hole states exhibited dramatically longer lifetimes [19]. Similar saturation of the ground states can be accomplished by doping the nanowire. For example, Wallentin et al. confirmed using PL in heavily doped WZ InP nanowires that the conduction band is in fact split as predicted theoretically by Pryor [14, 23].

The most sensitive and accurate technique using PL to measure excited states in single nanostructures is to use photoluminescence excitation (PLE) spectroscopy [15]. Here the emission intensity from a ground state or defect level is monitored as an excitation laser is tuned to higher energies. High quantum efficiency from the nanostructure is now often achieved and is vital for PLE as well as many applications. Thus when the laser is tuned into resonance with any excited state which is coupled to the ground or defect state, a strong increase in intensity will be seen. PLE does not directly measure the strength of the absorption into the excited state, but is convolved with the capture efficiency to the emitting state which is monitored. Indeed, if the excited state is not coupled to the monitored state at all, no increase in PL intensity would be observed even though significant excitation of electron-hole pairs results from the resonant excitation.

A more subtle effect is the fact that since the nanostructure can strongly couple to the electromagnetic field with strong polarization effects (particularly for nanowires), the strength of this coupling is also convolved in a PLE spectrum. Thus, while PLE spectra can show the energy position of excited states, making strong conclusions with respect to their relative intensities requires significant analysis.

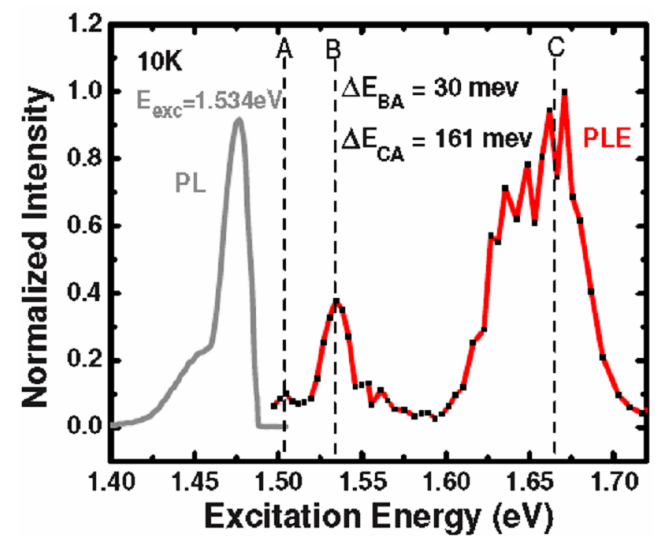

Fig. 1. Photoluminescence and photoluminescence excitation spectra from a single $100 \mathrm{~nm}$ diameter InP nanowire at $10 \mathrm{~K}$. PL spectrum is shown in grey with a peak at $1.47 \mathrm{eV}$, while the PLE spectrum is to the right with features at $1.50,1,54$ and $1.67 \mathrm{eV}$ associated with excitonic transitions associated with the $A$-, $B$ - and $C$-hole bands [13].
In Fig. 1 we show a PLE spectrum from a single-phase WZ InP nanowire [13]. Because of the hexagonal symmetry, crystal field effects and the spin-orbit interaction split the valence band at zone center into three identifiable energy states, the so-called $A, B$, and $C$ valence bands. In addition, the optical selection rules (isotropic for ZB InP nanowires) are also strongly affected because the $A$-valence band to conduction band transition is only allowed for the electric field polarized perpendicularly to the $\langle 111\rangle$ axis which lies along the nanowire [12]. Because the WZ InP nanowire was grown at very high $\mathrm{V} / \mathrm{III}$ ratio, arsenic antisite defects resulted in a strong defect emission about $50 \mathrm{meV}$ below the band edge. Using a sharp edge filter to remove the specularly scattered laser light, we monitored the intensity of the defect emission as the laser was tuned through the $A$-valence band to conduction band, $B$-to conduction band, and $C$-to conduction band excitation transitions. Clear intensity resonances were observed for all of these transitions, through the $A$-to conduction band transition was relatively weak as expected since the excitation is only allowed for perpendicularly polarized laser excitation. Through such measurements one can extract the crystal field and spin-orbit energies to be $52 \mathrm{meV}$ and $139 \mathrm{meV}$, respectively [13].

\section{Raman scattering}

When light interacts with materials in the Raman scattering, photons are scattered with energies associated with the creation or destruction of excitations of the material. These excitations frequently are associated with phonons, but features associated with electronic or spin excitations are also possible. In nanostructures the Raman scattering from phonons has been an effective tool for measuring the intrinsic strain in the nanostructure. For example, spatially dependent strains have been measured in CdS nanosheets [24] as well as the intrinsic strains involved in $\mathrm{GaAs} / \mathrm{GaP}$ core-shell structures where the $3.6 \%$ lattice mismatch results in a nearly $10 \%$ change in the direct band gap [8].

The Raman scattering with phonons can also be a sensitive measure of the electronic structure in nanostructures. Normally, the scattering efficiency of phonons is relatively weak (much weaker than photoluminescence, for example) but the strength of the scattering can be significantly enhanced if the incoming or outgoing photon is resonant with an intrinsic electronic excitation. In Fig. 2 the Raman scattering spectra from a single CdS nanowire associated with the creation of one longitudinal optical (LO) phonon is shown as a function of the incoming laser energy [25]. A clear resonant enhancement of the 1-LO Raman scattering efficiency is seen as the scattered light comes into resonance with the $A$-valence band to conduction band electronic transition. The lineshape of the enhancement was seen to have a width very similar to the PL emission linewidth from the same nanowire. A similar resonance was seen for the 2-LO scattering efficiency [25].

More recently, resonant Raman scattering was used as a sensitive probe of the band structure of wurtzite 


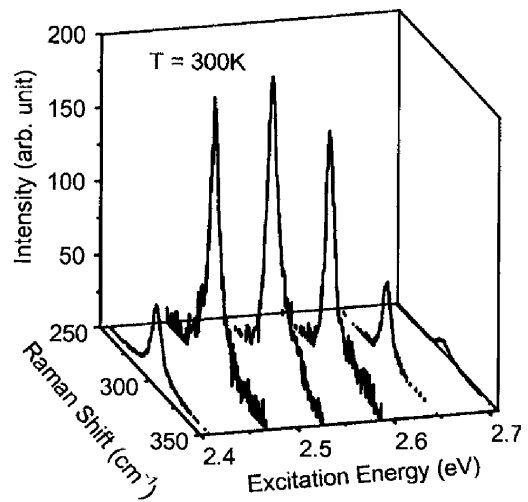

Fig. 2. Raman scattering spectra from a single CdS nanowire as a function of laser excitation energy. The peak results from creation of a single LO phonon. The underlying PL has been subtracted for clarity. Clear resonant enhancement of the 1-LO Raman spectra is seen when the outgoing scattered photon is in resonance with the CdS band edge [25].

GaAs nanowires which were grown by MBE using Ga-catalyzed growth [26]. As is seen in wurtzite InP (see Sect. 2 above), in WZ GaAs the valence band should split into $A$-, $B$ - and $C$-levels with energies determined by the crystal field and spin-orbit energies. Resonant Raman scattering showed that the $A$-to conduction band energy for WZ GaAs is, surprisingly, the same as the valence band to conduction band energy for ZB GaAs [26]. The $B$ - and $C$-valence band energies imply that the crystal field splitting was $189 \mathrm{meV}$ and the spin-orbit energy was $379 \mathrm{meV}$ in WZ GaAs [26].

\section{Photocurrent spectroscopy}

An extremely sensitive technique for measuring excited state energies in nanostructures is to measure the photocurrent as a function of optical excitation energy [27]. Since nanowires are nanoscaled only along two dimensions (the diameter of the nanowire) and the length of the wire is typically several $\mu \mathrm{m}$, it is straightforward to use lithographic methods to create metallic contacts at the end of the nanowires $[28,29]$. Frequently this is done using extremely high-resolution writing techniques like electron beam lithography, or even focused ion beam (FIB) deposition, but it is also possible to use standard photolithographic techniques using a shadow mask to create metallic contacts $[28,29]$.

Figure $3 \mathrm{a}$ shows optical and scanning electron microscopy (SEM) micrographs of a single InP nanowire which is contacted between two titanium-aluminum metal contacts. The pads are typically large enough to easily wire bond leads when the nanowire-device is mounted into a ceramic package. Most frequently without special preparation the metal contacts create back-to-back Schottky barriers across the nanowire, but with some effort it is possible to make good ohmic contacts to nanowires. In either case (ohmic or Schottky) such a nanoscale device makes an extremely sensitive detector for incoming light. When the incoming light has an energy above the gap energy it is possible to measure pho- tocurrents with sub- $\mu \mathrm{W}$ laser excitation powers. With a tunable laser the magnitude of the photocurrent as a function of laser energy provides a direct measure of the excited state structure within the nanowire. Unlike PLE (see the discussion above) as long as the optical excitation creates electron-hole pairs and any radiative or nonradiative recombination time is larger than the separation time of the electron-hole pair, the photocurrent is a good representation of the absorption strength. Let us note that photocurrent spectroscopy is still subject to the possibly strong interactions of the nanowire with the optical electromagnetic field which can create resonances and polarization effects.

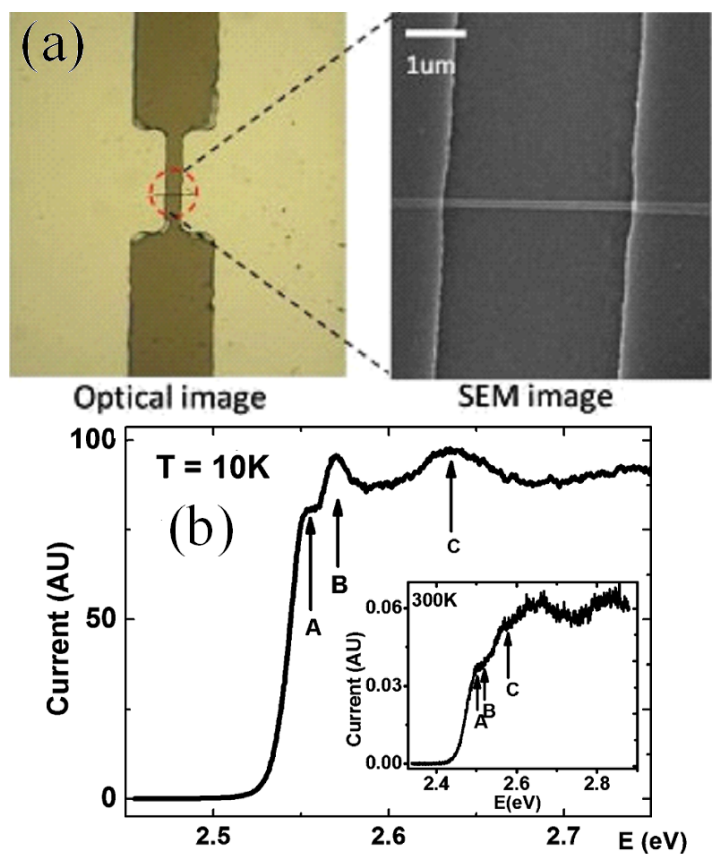

Fig. 3. (a) Top part shows optical and SEM image of a single InP nanowire two-terminal device with titaniumaluminum metallic contacts. (b) Photocurrent spectra from a CdS nanosheet device excited using a white light source filtered by a $1 / 8 \mathrm{~m}$ single grating spectrometer. Clear peaks associated with band-to-band transitions between the $A$-, $B$ - and $C$-valence bands and the conduction band are seen [29].

Recently, photocurrent spectra obtained from ZB and WZ InP single nanowire devices excited by a $\mathrm{CW}$ Ti-sapphire laser at room temperature showed distinct signatures at the band edges characteristic for these polytypes with the WZ band edge $80 \mathrm{meV}$ higher in energy than the corresponding ZB band edge [28]. The sensitivity of such nanodevices is so high that it is possible to excite measureable photocurrents even with a white-light source which is filtered by a spectrometer [29]. In Fig. 3b we show such a photocurrent spectrum at both room temperature (inset) and $10 \mathrm{~K}$ for a CdS nanosheet device. The average power for the filtered white light was in the several nanowatt range, yet structures for band- 
-to-band transitions between the $A$-, $B$ - and $C$-valence bands to the conduction band were easily seen [29]. Resonant peaks at these transitions result from excitonic enhancements to the oscillator strength for absorption. Kumar et al. also were able to observe nonlinear two-photon excitation in these structures by exciting with very low power Ti-sapphire infrared laser pulses [29]. Because the selection rules for nonlinear two-photon excitation are sensitive to the symmetry of the electronic bands, it is expected that two-photon excited spectral currents should also provide significant information on nanostructure excitations.

Photocurrent spectra from single nanostructures can provide significant information, particularly when the nanomaterial does not show strong PL or when the emission is in the infrared where both laser sources and photodetectors are lacking. Photocurrent spectroscopy can also provide information on more complicated structures such as for an InAsP quantum dot embedded within an InP nanowire as recently shown by van Kouwen et al. [21]. Extremely narrow resonances were seen which were characteristic of the quasi-zero-dimensional density of states expected for the quantum dot.

\section{Photomodulated Rayleigh scattering}

With macro-materials (even 2D epilayers or quantum wells) absorption or reflection of light can be a very sensitive technique for probing electronic structure. However, nanostructures are usually nanoscaled along at least two dimensions and so this creates several significant difficulties. Chief among these challenges is that the diffraction limit of light means that light can be focused at best only to a $\mu \mathrm{m}$ or so, and so the nanostructure may only be a small fraction of the focused spot. As a result, reflected or transmitted light is completely dominated by light which does not interact with the nanostructure. However, many nanostructures do interact strongly light which can strongly affect both the scattering efficiency and also the polarization of the scattered light.

Semiconductor nanowires strongly interact with the electromagnetic field. A number of papers illuminate the effect on the excitation and emission of PL which can have a dramatic effect on polarization [30-32]. In addition, the polarization of the light scattered by semiconductor nanostructures is also strongly affected [33] and was described mathematically in the late nineteenth century by lord Rayleigh [34]. For nanowires of diameter less than $90 \mathrm{~nm}$ the scattered light for nearly all energies is strongly polarized parallel to the nanowire long axis. For larger diameter nanowires, it is possible to have strong scattered light polarized either parallel or perpendicular to the long axis, or it can be unpolarized. Because the scattered light is so strongly polarized, one can use this to detect only the light which interacts with the nanostructure. Using a photoelastic modulator the polarization of a tunable probe beam can be modulated between parallel and perpendicular to the nanowire axis at $100 \mathrm{kHz}$ (see the schematic diagram in Fig. 4a) [35]. By using a balanced detector and a lock-in amplifier one can monitor

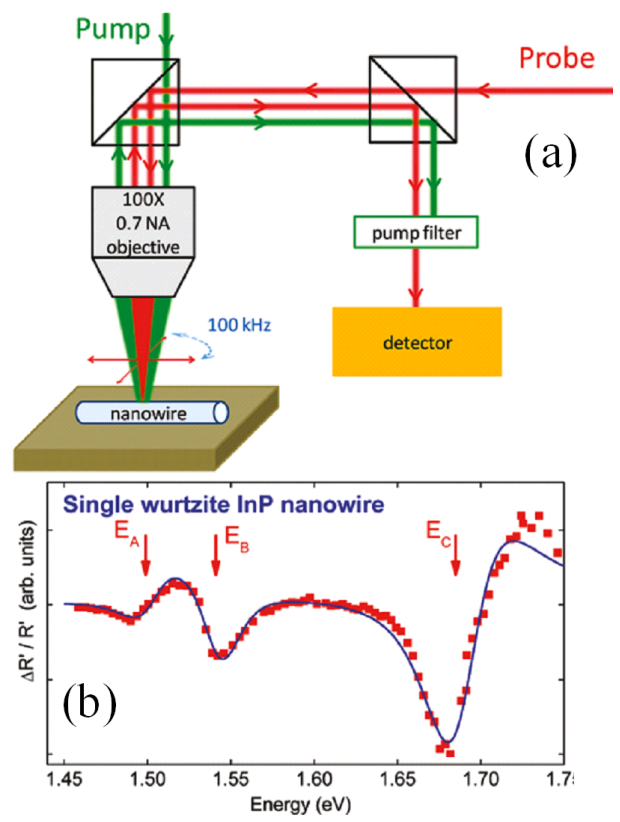

Fig. 4. (a) Schematic diagram showing the photomodulated Rayleigh scattering experimental technique. A $532 \mathrm{~nm}$ pump laser excites carriers into a single semiconductor nanowire. A tunable Ti-sapphire probe laser is focused onto the nanowire while the polarization is oscillated between parallel and perpendicular to the NW axis at $100 \mathrm{kHz}$ and the scattered light is detected by a balanced detector. (b) Typical PMRS spectrum from a $100 \mathrm{~nm}$ diameter WZ InP nanowire. Derivative features are seen corresponding to optical transitions associated with the $A$-, $B$ - and $C$-valence bands [35].

directly the light which interacts with the nanowire since the substrate does not exhibit strong polarization effects.

Because the scattered light efficiency and relative polarization depends only on the radius of the nanowire and the dielectric mismatch between the nanowire and its surroundings, the spectral dependence of the scattering efficiency is fairly featureless (particularly for small diameter wires) [35]. To bring out the electronic structure of single nanowires, one can use a pump laser which excites electron-hole pairs high up into the bands which modulates the band gap slightly. Using an optical chopper at several hundred $\mathrm{Hz}$ to modulate the pump beam, one is able to detect the change in the scattered light intensity and polarization using a second lock-in amplifier tuned to the chopper frequency. Recently, we have shown that by such photomodulation of the Rayleigh scattering (PMRS) one can get derivative spectra which display directly the energies of the fundamental excitations in the nanowires [35]. In Fig. 4b we show a typical photomodulated Rayleigh scattering spectrum from a $100 \mathrm{~nm} \mathrm{WZ}$ InP nanowire [35]. Narrow derivative features are seen for optical transitions from the $A-, B$ - and $C$-valence bands to the conduction band. Montazeri et al. have shown that PMRS spectra can resolve all of the major band-to-band excitations for $\mathrm{ZB}$ and $\mathrm{WZ}$ InP as well as $\mathrm{ZB}$ GaAs with excellent signal to noise at both $300 \mathrm{~K}$ and $10 \mathrm{~K}$ [35]. 
Because PMRS spectroscopy involves the interaction of a single nanostructure with both a pump and probe laser, it is possible to extend the technique to measure the dynamics of electron-hole pairs photoexcited by a fast pump pulse using the scattered light from a delayed fast probe pulse. The spatial resolution of the technique is only limited by the ability to focus the pump and probe beams which could be significantly enhanced using a solid immersion lens.

\section{Summary}

With the ever increasing ability to tune the physical structure and confined electronic states within a nanostructure, the ability to sensitively measure these states within a single nanostructure will also become increasingly important. Here, we have reviewed a number of optical techniques that can illuminate the electronic excitations within such single nanostructures.

\section{Acknowledgments}

We acknowledge the financial support of the $\mathrm{Na}$ tional Science Foundation through grants DMR-0806700, 0806572, 1105362, 1105121, and ECCS-1100489, and the Australian Research Council. The Australian National Fabrication Facility is acknowledged for access to the growth facilities used in this research.

\section{References}

[1] M. Law, J. Goldberger, P.D. Yang, Ann. Rev. Mater. Res. 34, 83 (2004).

[2] Y.N. Xia, P.D. Yang, Y.G. Sun, Y.Y. Wu, B. Mayers, B. Gates, Y.D. Yin, F. Kim, Y.Q. Yan, Adv. Mater. 15, 353 (2003).

[3] E.C. Garnett, M.L. Brongersma, Y. Cui, M.D. McGehee, Ann. Rev. Mater. Res. 41, 269 (2011).

[4] P. Caroff, J. Bolinsson, J. Johansson, IEEE J. Sel. Top. Quant. Electron. 17, 829 (2011).

[5] H.J. Joyce, Q. Gao, H.H. Tan, C. Jagadish, Y. Kim, J. Zou, L.M. Smith, H.E. Jackson, J.M. Yarrison-Rice, P. Parkinson, M.B. Johnston, Prog. Quant. Electron. 35, 23 (2011).

[6] S. Barth, F. Hernandez-Ramirez, J.D. Holmes, A. Romano-Rodriguez, Prog. Mater. Sci. 55, 563 (2010).

[7] D. Narducci, Sci. Adv. Mater. 3, 426 (2011).

[8] M. Montazeri, M. Fickenscher, L.M. Smith, H.E. Jackson, J. Yarrison-Rice, J.H. Kang, Q. Gao, H.H. Tan, C. Jagadish, Y. Guo, J. Zou, M. Pistol, C.E. Pryor, Nano Lett. 10, 880 (2010).

[9] R. Agarwal, Small 4, 1872 (2008).

[10] M.E. Pistol, C.E. Pryor, Phys. Rev. B 78, 115319 (2008).

[11] M.E. Pistol, C.E. Pryor, Phys. Rev. B 80, 035306 (2009).

[12] A. Mishra, L.V. Titova, T.B. Hoang, H.E. Jackson, L.M. Smith, J.M. Yarrison-Rice, Y. Kim, H.J. Joyce, Q. Gao, H.H. Tan, C. Jagadish, Appl. Phys. Lett. 91, 263104 (2007).

[13] S. Perera, K. Pemasiri, M.A. Fickenscher, H.E. Jackson, L.M. Smith, J. Yarrison-Rice, S. Paiman, Q. Gao, H.H. Tan, C. Jagadish, Appl. Phys. Lett. 97, 023106 (2010).
[14] A. De, C.E. Pryor, Phys. Rev. B 81, 155210 (2010).

[15] G.D. Gilliland, Mater. Sci. Eng. R: Reports $R$ 18, 99 (1997).

[16] K.P. Hewaparakrama, A. Wilson, S. Mackowski, H.E. Jackson, L.M. Smith, G. Karczewski, J. Kossut, Appl. Phys. Lett. 85, 5463 (2004).

[17] T.B. Hoang, L.V. Titova, J.M. Yarrison-Rice, H.E. Jackson, A.O. Govorov, Y. Kim, H.J. Joyce, H.H. Tan, C. Jagadish, L.M. Smith, Nano Lett. 7 , 588 (2007).

[18] H. Rho, K.Y. Lee, T.B. Hoang, L.V. Titova A. Mishra, L.M. Smith, H.E. Jackson, J.M. Yarrison-Rice, Y.J. Choi, K.J. Choi, J.G. Park, Appl. Phys. Lett. 92, 013111 (2008).

[19] K. Pemasiri, M. Montazeri, R. Gass, L.M. Smith, H.E. Jackson, J. Yarrison-Rice, S. Paiman, Q. Gao, H.H. Tan, C. Jagadish, X. Zhang, J. Zou, Nano Lett. 9, 648 (2009).

[20] J.M. Bao, D.C. Bell, F. Capasso, J.B. Wagner, T. Martensson, J. Tragardh, L. Samuelson, Nano Lett. 8, 836 (2008).

[21] M.P. van Kouwen, M.E. Reimer, A.W. Hidma, M.H.M. van Weert, R.E. Algra, E. Bakkers, L.P. Kouwenhoven, V. Zwiller, Nano Lett. 10, 1817 (2010).

[22] L.M. Smith, H.E. Jackson, J. Yarrison-Rice, C. Jagadish, Semicond. Sci. Technol. 25, 024010 (2010).

[23] J. Wallentin, K. Mergenthaler, M. Ek, L.R. Wallenberg, L. Samuelson, K. Deppert, M. Pistol, M.T. Borgstrom, Nano Lett. 11, 2286 (2011).

[24] M. Montazeri, L.M. Smith, H.E. Jackson, J.M. Yarrison-Rice, Y.J. Choi, J.G. Park, Appl. Phys. Lett. 95, 083105 (2009).

[25] A. Abdi, L.V. Titova, L.M. Smith, H.E. Jackson J.M. Yarrison-Rice, J.L. Lensch, L.J. Lauhon, Appl. Phys. Lett. 88, 043118 (2006).

[26] B. Ketterer, M. Heiss, E. Uccelli, J. Arbiol, A. Fontcuberta i Morral, Acs Nano 5, 7585 (2011).

[27] C. Soci, A. Zhang, X. Bao, H. Kim, Y. Lo, D. Wang, J. Nanosci. Nanotechnol. 10, 1430 (2010).

[28] A. Maharjan, K. Pemasiri, P. Kumar, A. Wade, L.M. Smith, H.E. Jackson, J.M. Yarrison-Rice, A. Kogan, S. Paiman, Q. Gao, H.H. Tan, C. Jagadish, Appl Phys. Lett. 94, 193115 (2009).

[29] P. Kumar, A. Wade, L.M. Smith, H.E. Jackson, J.M. Yarrison-Rice, Y.J. Choi, J.G. Park, Appl. Phys. Lett. 98, 143102 (2011).

[30] H.E. Ruda, A. Shik, Phys. Rev. B 72, 115308 (2005).

[31] H.E. Ruda, A. Shik, J. Appl. Phys. 100, 024314 (2006).

[32] J.F. Wang, M.S. Gudiksen, X.F. Duan, Y. Cui, C.M. Lieber, Science 293, 1455 (2001).

[33] D. Zhang, J. Wu, Q. Lu, H.R. Gutierrez, P.C. Eklund, Nanotechnology 21, 315202 (2010).

[34] H.J.W. Strutt, Philos. Mag. 41, 107 (1871).

[35] M. Montazeri, A. Wade, M. Fickenscher, H.E. Jackson, L.M. Smith, J. Yarrison-Rice, Q. Gao, H.H. Tan, C. Jagadish, Nano Lett. 11, 4329 (2011). 